Canadian University Music Review

Revue de musique des universités canadiennes

\title{
Franz Schubert : un Pas Vers l'Atonalité
}

\section{Mario Leblanc}

Volume 9, numéro 2, 1989

URI : https://id.erudit.org/iderudit/1014907ar

DOI : https://doi.org/10.7202/1014907ar

Aller au sommaire du numéro

\section{Éditeur(s)}

Canadian University Music Society / Société de musique des universités canadiennes

\section{ISSN}

0710-0353 (imprimé)

2291-2436 (numérique)

Découvrir la revue

Citer cet article

Leblanc, M. (1989). Franz Schubert : un Pas Vers l’Atonalité. Canadian

University Music Review / Revue de musique des universités canadiennes, 9(2),

84-115. https://doi.org/10.7202/1014907ar

All Rights Reserved (C Canadian University Music Society / Société de musique des universités canadiennes, 1989
Ce document est protégé par la loi sur le droit d'auteur. L'utilisation des services d'Érudit (y compris la reproduction) est assujettie à sa politique d'utilisation que vous pouvez consulter en ligne.

https://apropos.erudit.org/fr/usagers/politique-dutilisation/ 


\title{
FRANZ SCHUBERT : UN PAS VERS L'ATONALITÉ
}

\author{
Mario Leblanc
}

On reconnaît généralement à Schubert une écriture tonale particulière. ${ }^{1}$ Il s'agit, en fait, d'une caractéristique essentielle de son langage. Curieusement toutefois, cet aspect semble passer en second lorsqu'on examine les textes sur Schubert : on préfère trop souvent retracer l'influence beethovenienne - au plan formel, notamment -, ce qui a bien peu d'importance si ce que nous entendons est typiquement schubertien. À vrai dire, l'écriture harmonique de Schubert est à proprement parler révolutionnaire, et, tel que le suggère le titre de cet article, on peut même y trouver les prémisses de la destruction de la tonalité. Un des procédés mis en cuvre par le compositeur pour arriver à cette fin est le recours systématique à la division de l'octave en parties égales, et cela, à tous les niveaux du discours, tant macroscopiques que microscopiques.

\section{La division de l'octave en parties égales}

Suite à la théorie de Werckmeister, l'échelle tempérée égale repose sur une division rigoureuse de l'octave en 12 demi-tons égaux. Mais le tempérament égal permet aussi d'envisager d'autres modalités de division de l'octave en intervalles égaux, constitués d'un nombre entier de demi- tons. L'octave est ainsi divisible en :

2 tritons, orthographiés comme quarte augmentée ou quinte diminuée (ex. : do-fa\#-do);

3 tierces majeures, une pouvant être orthographiée comme quarte diminuée (ex. : do-mi-lab-do);

4 tierces mineures, une pouvant être orthographiée comme seconde augmentée (ex. : do-mib-fa\#-la-do);

1 M.J. Brown écrit : "He was a pioneer for new, far-reaching harmonic and tonal experiments." (1980:778) 
6 tons, un pouvant être orthographié comme tierce diminuée, soit la gamme par tons. ${ }^{2}$

Nous devons ici faire remarquer le caractère "étranger" que possède au premier abord, du moins - le principe de la division parfaitement symétrique de l'octave par rapport aux habitudes tonales. Rappelons, en effet, que les gammes majeure et mineure, fondements de la tonalité, se présentent, respectivement, comme une combinaison plus ou moins symétrique de tons et de demi-tons. $\mathrm{D}^{\prime}$ autre part, dans les œuvres relevant du système tonal, les déplacements par tierces, qu'ils soient ascendants ou descendants, $s^{\prime}$ effectuent le plus souvent par alternance d'intervalles majeurs et mineurs; on note aussi que les enchaînements $d^{\prime}$ accords se font majoritairement par quinte ou quarte justes.

Malgré ces pratiques, toutefois, on ne saurait oublier que la tonalité admet des structures de parfaite symétrie au plan de l'écriture verticale, et cela, pour certains accords ayant fonction de dominante. Ainsi :

la division de l'octave en tierces mineures donne un accord dit de septième diminuée (que nous interprétons comme accord de neuvième mineure de dominante, avec fondamentale tacite);

la division de l'octave en tierces majeures donne un accord dit de quinte augmentée;

le triton constitue un intervalle important de l'accord septième de dominante, où il loge entre la sensible et la sous-dominante de la tonalité, les deux notes qui créent tension dans l'accord concerné;

la gamme par tons elle-même peut figurer tout entière dans un accord de dominante particulièrement riche, composé des fondamentale, tierce, quinte doublement altérée (c'est-à-dire haussée et abaissée à la fois), septième mineure et neuvième majeure.

2 La gamme par tons est l'une des gammes à transpositions limitées relevées par Messiaen. Schubert emploiera une autre de ces gammes, la gamme dite "octatonique", composée de l'alternance "ton, demi-ton, ton, demi-ton, etc." dans la Sonate en do majeur, D. 840 (1825), et cela, dans un contexte tout à fait comparable au début de la Sonate no 9 de Scriabine, écrite quelque cent ans plus tard (1915). (Voir exemple 1.) 


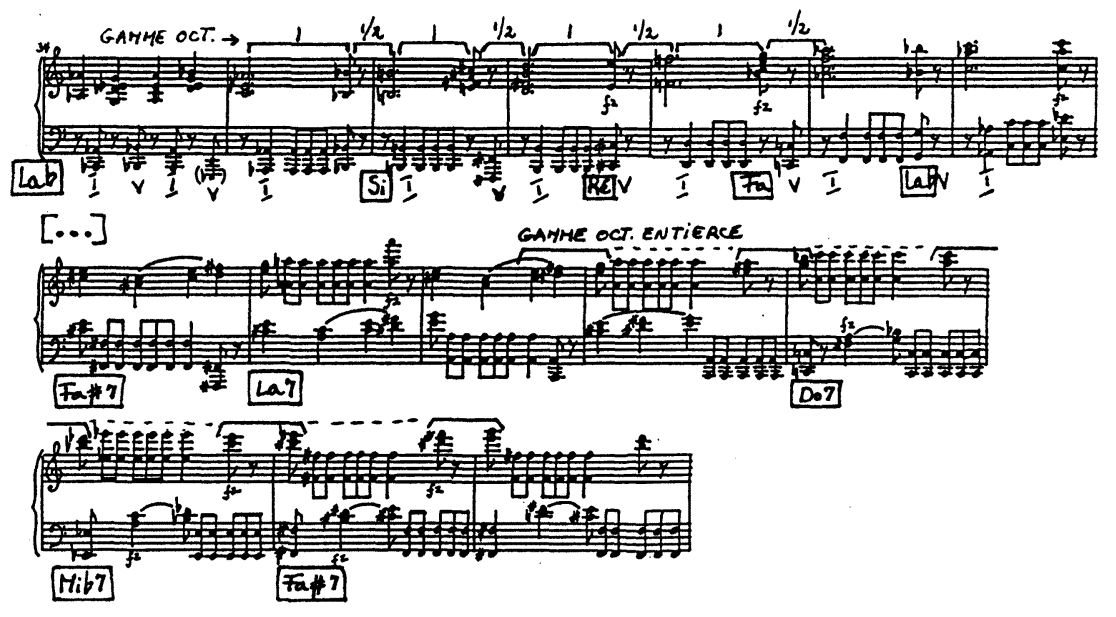

Exemple 1a): Schubert : Sonate en Do, D.840, I

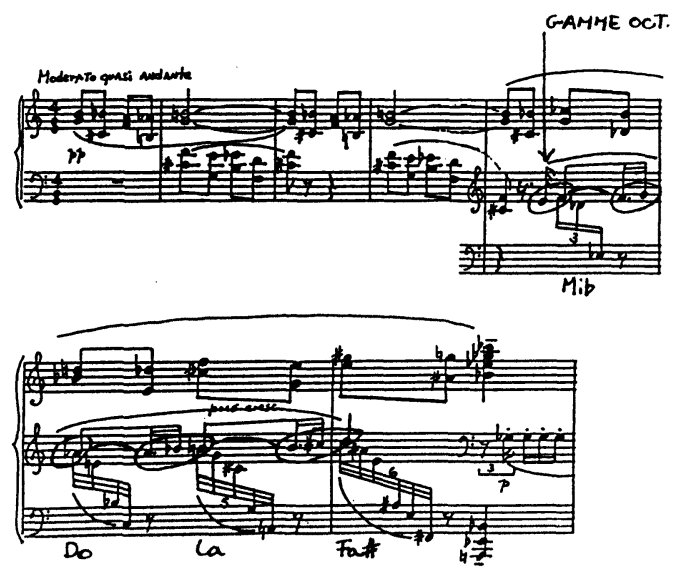

Exemple 1b) : Scriabine : Sonate no 9, op. 68, début 
Or, si l'on applique le procédé de la division de l'octave en parties égales à des fondamentales d'accords parfaits (majeurs ou mineurs), on s'aperçoit que l'opération est susceptible de mettre en jeu de multiples niveaux, incluant aussi bien le macroscopique que le microscopique. En effet, une fondamentale d'accord parfait peut être considérée :

à l'échelle d'un simple accord;

à l'échelle d'un enchaînement harmonique : comme pôle d'un accord de tonique secondaire ou encore, de dominante sur laquelle il y a insistance;

à l'échelle d'une section de mouvement : comme pôle d'une tonalité provisoire ou pôle d'une tonalité durable (la tonalité d'un thème, par exemple);

à l'échelle d'un mouvement entier;

à l'échelle de la totalité d'une œuvre en plusieurs mouvements.

Précisons que l'analyse harmonique d'un enchaînement de fondamentales séparées, par exemple, d'une tierce majeure ne va pas toujours de soi. Prenons pour illustration les accords consécutifs Do-Lab-Mi-Do. On peut certes identifier, par rapport au ton de Do "mixte" (c'est-à-dire qui amalgame les modes majeur et mineur), que $L a b$ majeur a pour fonction VI, et que Mi est III (en supposant la triade mineure), mais l'enchaînement I-VIb-III-I est fort peu courant et son sens tonal apparaît flou.

En fait, le caractère non-tonal de ce type d'enchaînement est important, et il représente une particularité de la division de l'octave en parties égales. Confrontons-le à un enchaînement d'accords en relation de tierces diatoniques :

triades en relation de tierces diatoniques (ex. : Do-La-Fa-Ré-SiSol-Mi-Do) : nous nous trouvons face à un enchaînement jugé faible, à cause des deux notes communes entre chaque couple de triades. La conséquence, dans un cercle tonal (ou cycle des quintes), est un va-et-vient concentré dans une région du cercle (voir exemple 2a); 
Exemple 2a)

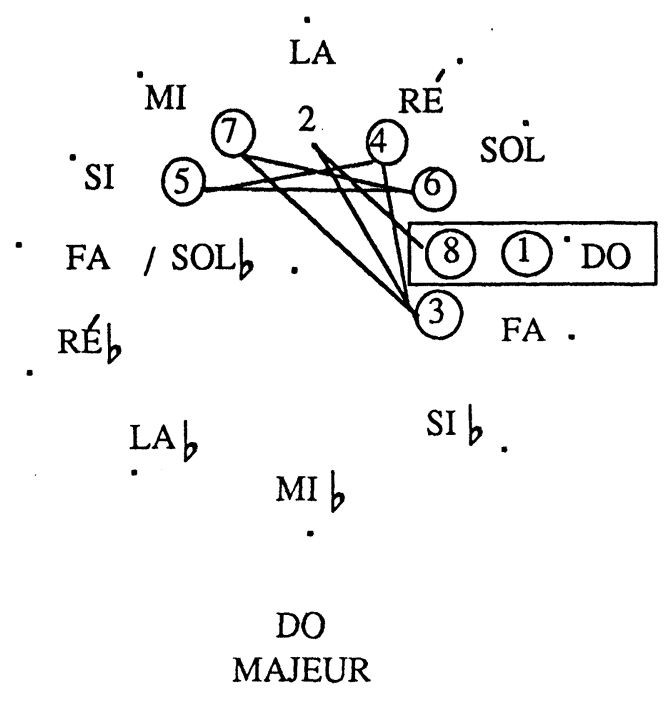

TIERCES DIATONIQUES

DO-LA-FA-RÉ-SI-SOL-MI-DO

Exemple 2b)

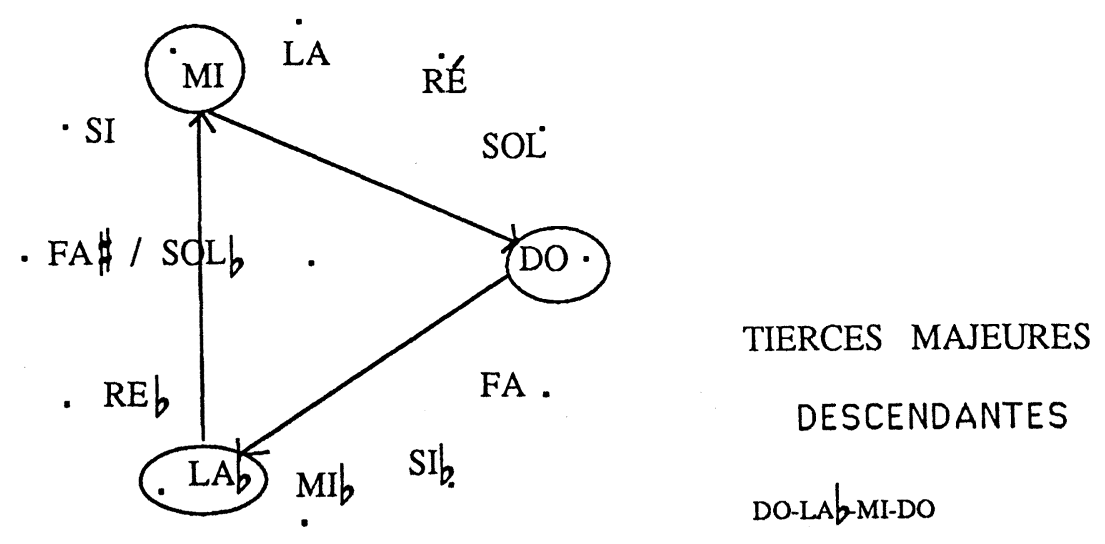


triades séparées par des tierces de même nature (ex. : Do-Lab$M i-D o)$ : dans un cercle tonal, la conséquence est celle d'un tour complet (voir exemple 2b). Il semble ici que "plus on s'éloigne, plus on se rapproche". La tonalité est comprise de façon circulaire, grâce à des considérations d'ordre enharmonique.

Schubert utilise, de manière privilégiée, la division de l'octave en tierces majeures descendantes, ce qui rend possible le tour du cercle tonal dans le sens authentique. En comparaison, on ne trouve, dans sa production, que de rares exemples de tierces majeures ascendantes. Le présent article insiste donc sur différents niveaux d'emploi de la division de l'octave en trois parties égales - $c^{\prime}$ est ce qu'on trouvera au point $3-,{ }^{3}$ mais il rend compte aussi d'autres types de divisions utilisés par Schubert : divisions en tierces mineures, en tons et en tritons - ce qu'on trouvera au point 2 .

Le recours par Schubert à la division de l'octave en parties égales a été partiellement mis en lumière par certains théoriciens. ${ }^{4}$ Elmar Seidel (1963) est celui qui va plus loin en ce sens, lorsqu'il traite de l'enharmonie; il donne plusieurs exemples de division symétrique de l'octave dans l'œuvre schubertien, entrant tantôt dans des explications de fond, se contentant ailleurs d'une simple mention. D'autres musicologues ont plutôt "erré" dans leurs recherches, puisque la paternité du procédé est attribuée tour à tour à Liszt, Brahms, Chopin, Rossini, Wolf et même Bartók..$^{5}$ A vrai dire, on trouve des traces de ce

3 Pour une étude en détail de ce sujet, cf. Leblanc (1985).

4 Cf. Seidel (1963), Federhofer (1978), Webster (1978), Badura-Skoda (1982), Taruskin (1985).

5 Un bref survol : Carl Dalhaus (1980:183) attribue le procédé à Bartók, qui développerait ainsi des idées explorées par Liszt; R.F. Goldman (1965) mentionne également le nom de Liszt pour la division en tierces descendantes, avançant celui de Wolf pour la division en tierces ascendantes; Aldwell et Sachter trouvent "ambitieuse" la succession des tonalités par tierces mineures d'un mouvement de la Quatrième Symphonie de Tchaikovski (1979 : 247), mais ils incluent tout de même un exemple de Schubert, basé sur une succession de tierces majeures, avec d'autres de Rossini et de Liszt (ibid. : ex. 31-26 à 31-28). 
procédé chez Beethoven, ${ }^{6}$ voire, chez Mozart. ${ }^{7}$ Mais c'est Schubert qui est le premier à en faire un usage systématique, et cela, à toutes les époques de sa production. Les exemples existent à foison, et ceux que nous présentons ci-dessous ne constituent qu'un mince échantillonnage, puisé dans des œuvres de différents genres et de différentes périodes.

\section{La division de l'octave par tons, tritons et tierces mineures}

Chacune de ces divisions mériterait que l'on s'y arrête, spécialement la division par tierces mineures. ${ }^{8}$ Par mesure d'économie d'espace cependant, nous nous en tiendrons ici à un exemple unique, mais qui a l'avantage de réunir les trois types de division. Il s'agit du développement du premier mouvement de la Sonate en Do majeur, $D$. 279 (1815) - voir exemple 4.

Le mouvement est en Do majeur, et l'exposition se termine en Sol majeur; cette exposition ne comporte pas de surprise sur le plan harmonique. Le développement débute (mes. 86) par une marche harmonique modulante, dans laquelle l'accord de Ré majeur, ayant fonction de $\mathrm{V}$ en Sol, devient sixte napolitaine en Do\# mineur (mes. 89). ${ }^{9}$ Cet accord est suivi de la dominante de Do\#, présentée en deux étapes $\left[\mathrm{I}_{4}^{6}-\mathrm{V}\right](\mathrm{m} .90)$ et constituant le modèle $\mathrm{d}^{\prime}$ une marche qui se déplace par secondes descendantes : nous avons donc $S i$ mineur (m. 91), La mineur (m. 92) et Sol mineur (m. 93). On demeure sur place,

6 Schenker signale que les pôles du développement du premier mouvement de la Sonate Appassionata, op. 57 (1804) sont en rapport de tierces majeures (1979 : ex. 114-8). Il ne parle cependant pas de la présence d'un procédé similaire dans la Sonate pour violon et piano, op. 24, du même Beethoven, se limitant, dans ce cas, à des considérations d'ordre enharmonique (ibid. : ex. 100-6b).

7 À titre de référence, cf. le début du développement du dernier mouvement de la Symphonie no 39, en Mi bémol, K. 543 (voir exemple 3).

8 Une illustration probante de l'usage de ce procédé à différents niveaux est le premier mouvement de la Sonate en Do majeur, "Reliquie", D. 840, mouvement auquel nous avons déjà fait référence en parlant de la gamme "octatonique" (cf. exemple 1).

9 Dans notre texte, les notes impliquées dans le processus de la division de l'octave en parties égales seront identiquées en italiques. 

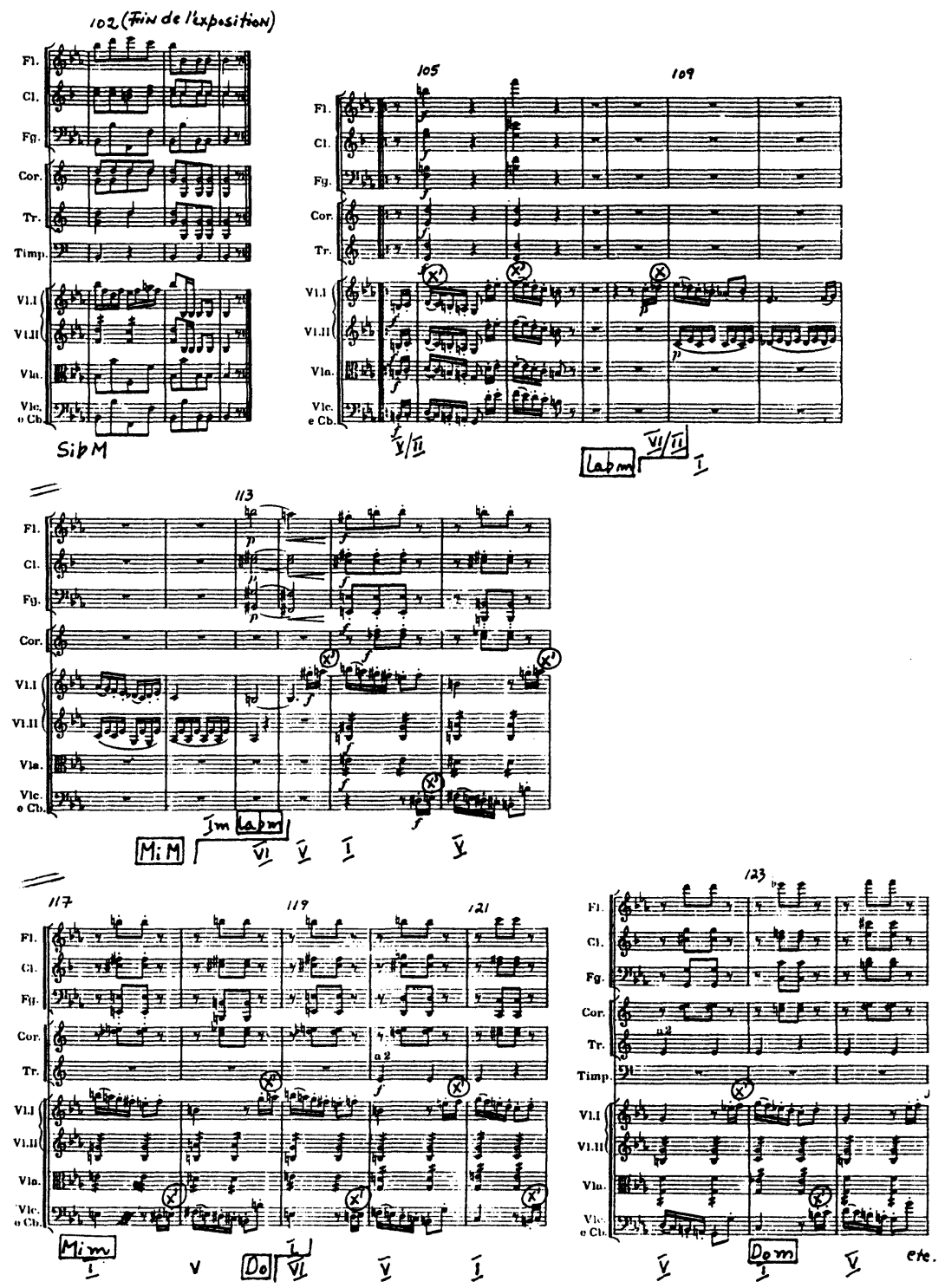

Exemple 3 : Mozart : Symphonie no 39 en Mi , IV 

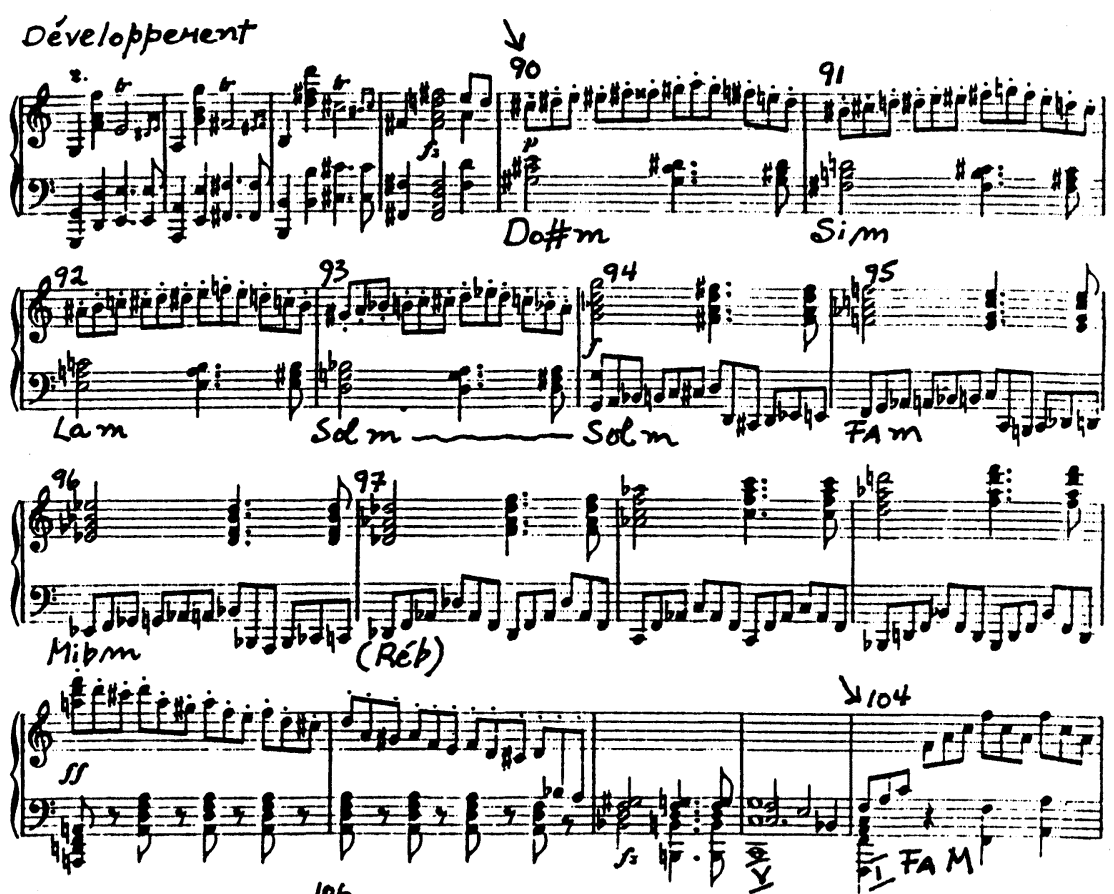

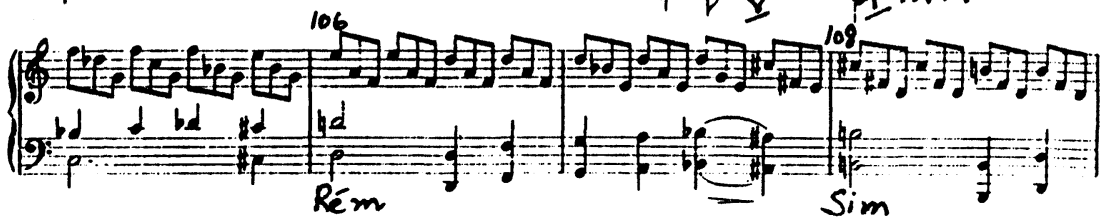

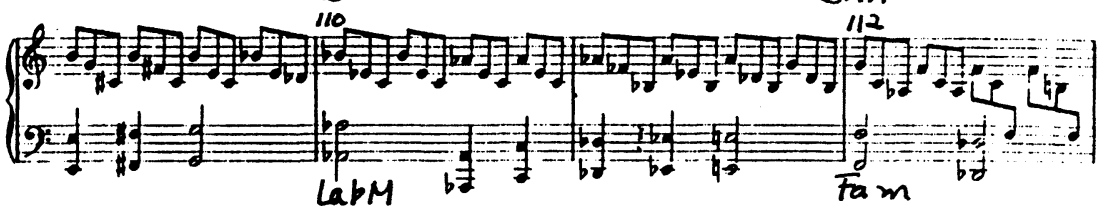
(4)

Exemple 4 : Schubert : Sonate en Do, D. 279, I 
et par le jeu de l'inversion des voix, la fonction $\mathrm{V}$ sous la forme [ $\left.\mathrm{I}_{4}^{\prime}-\mathrm{V}\right]$ se dédouble en deux fonctions, I-V, (m. 94) pour un nouveau modèle de marche, toujours par tons descendants : $F a$ mineur (m. 95), Mib mineur (m. 96); on aboutit enfin à un accord de Réb majeur (m. 97) qui annonce l'arrivée prochaine de $\mathrm{Fa}$ majeur (m. 104).

Nous avons ici une gamme par tons complète dont chacun des éléments est une tonique secondaire : Do\#-Si-La-Sol-Fa-Mib-Réb. Le fait que ces toniques soient mineures ajoute à la hardiesse des enchaînements, et on peut noter au passage que Schubert évite la redite du Do\# mineur de départ par l'emploi de Réb majeur.

La division de l'octave en deux tritons est également présente ici : la gamme par tons a pour pôles Do\#-Réb, notes enharmoniques qui se trouvent justement à distance de triton de Sol, tonalité de la fin de l'exposition et du début du développement. De plus, cette gamme par tons (Do\# à Réb) ne subit qu'une seule halte : sur Sol mineur (m. 93-94) : on y demeure deux fois plus longtemps que sur les autres toniques secondaires. Schubert a certainement voulu souligner le centre de la gammes par tons.

À partir de la mesure 104 du même exemple, on trouve une utilisation de la division de l'octave en tierces mineures. Les éléments de cette division sont à nouveau des toniques secondaires en marche harmonique. Le modèle de la marche est l'enchaînement harmonique I-V-V/VI-VI (m. 104 jusqu'au début de m. 106), soit le déplacement d'un accord de tonique majeur vers son relatif mineur, lequel se trouve justement à distance de tierce mineure. Schubert évite la version diatonique de cette marche et, profitant de l'ambiguité de l'accord septième diminuée, introduit plutôt une nouvelle application de division symétrique qui n'est pas sans dérouter l'auditeur. On part donc de $F a$ majeur (m. 104) pour aller successivement à $R e ́$ mineur (m. 106), puis, non sans surprise, vers $S i$ mineur (m. 108) et Lab majeur (m. 110), avant de revenir à $F a$, maintenant mineur (m. 112). Avec Fa-Ré-Si-Lab-Fa, nous avons bel et bien une division de l'octave en 4 tierces mineures.

On peut remarquer que Schubert n'élabore pas sa marche mécaniquement, puisque les tonalités majeures et mineures $n^{\prime}$ alternent pas : on a Majeur, mineur, puis l'inverse, mineur, Majeur. De plus, le compositeur évite l'identité parfaite entre les points de départ et d'arrivée de cette structure symétrique : Fa est majeur au départ, et mineur à l'arrivée. (Nous avons constaté le même phénomène pour les Do\#-Réb de la gamme par tons observée plus haut.) 
Cet exemple est assez particulier pour plusieurs raisons. Outre le fait que ce soit une auvre de jeunesse, il s'agit d'un spécimen - très rare où deux types différents de division de l'octave en parties égales se voisinent presqu'immédiatement. C'est aussi un des seuls exemples où la gamme par tons est employée autrement que d'une façon mélodique. Enfin, il faut bien dire que, vu la sagesse de l'exposition sur le plan tonal, la surprise engendrée par l'aspect non-tonal du développement est totale, le nombre de répétitions des modèles des marches ne réussissant pas à l'atténuer.

\section{La division de l'octave par tierces majeures}

Comme il a été mentionné plus haut, la division de l'octave par tierces majeures a été employée plusieurs fois et de diverses façons par Schubert. Nous en illustrerons ici plusieurs niveaux d'application, allant du macroscopique au microscopique.

\subsection{Tonalités des mouvements d'une auvre}

Prenons la Messe en Lab, D. 678 (1819-23) - voir exemple 5. Les tonalités des différents mouvements sont les suivantes :

- Kyrie

- Gloria

- Credo

- Sanctus et Hosanna

Benedictus

- Agnus Dei

Dona nobis Pacem
Lab majeur

$M i$ majeur

Do majeur

$\mathrm{Fa}$ majeur

Lab majeur

$\mathrm{Fa}$ mineur

Lab majeur 
Exemple 5 : Schubert : Messe en Lab majeur, D. 678, début des différentes parties
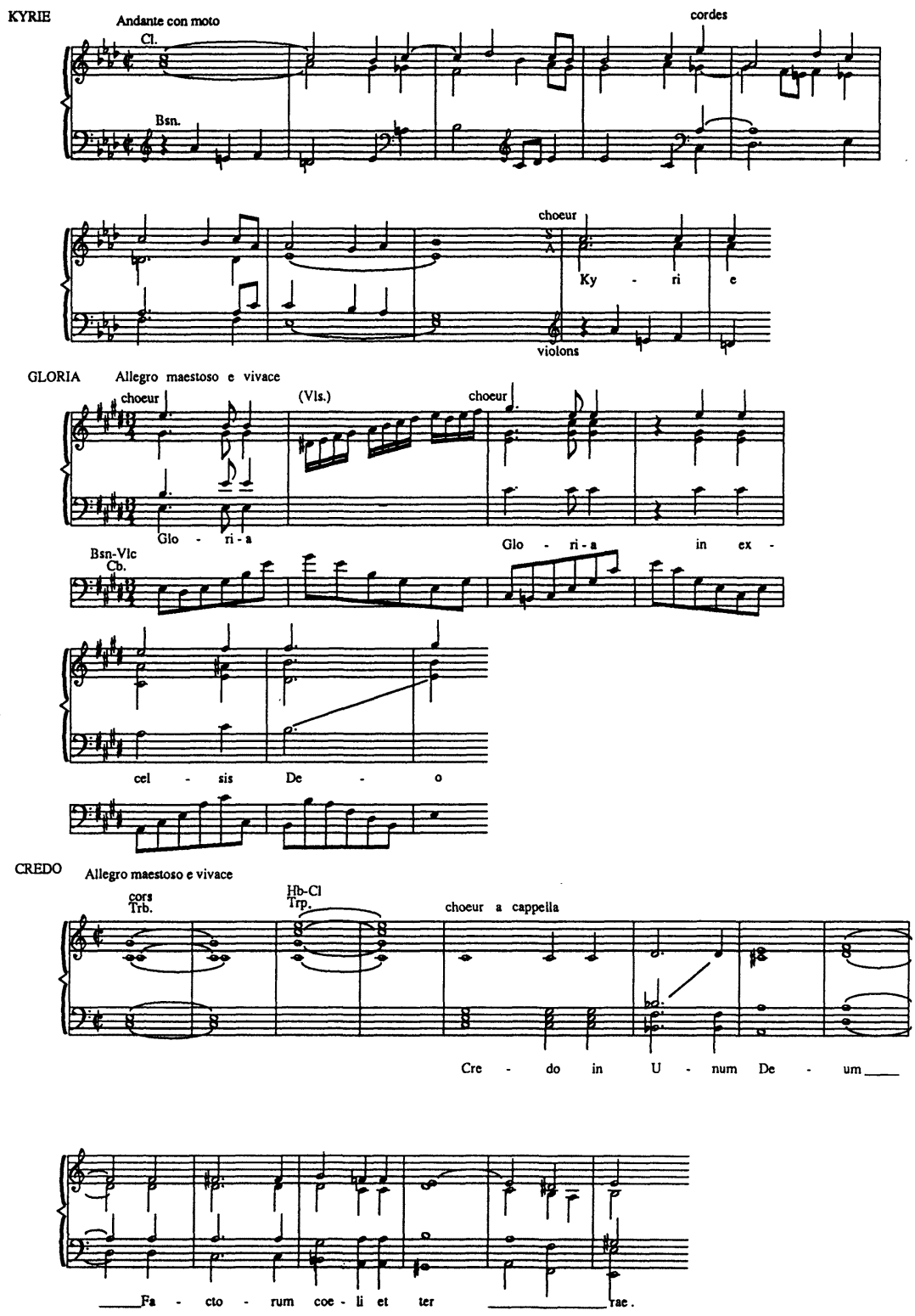

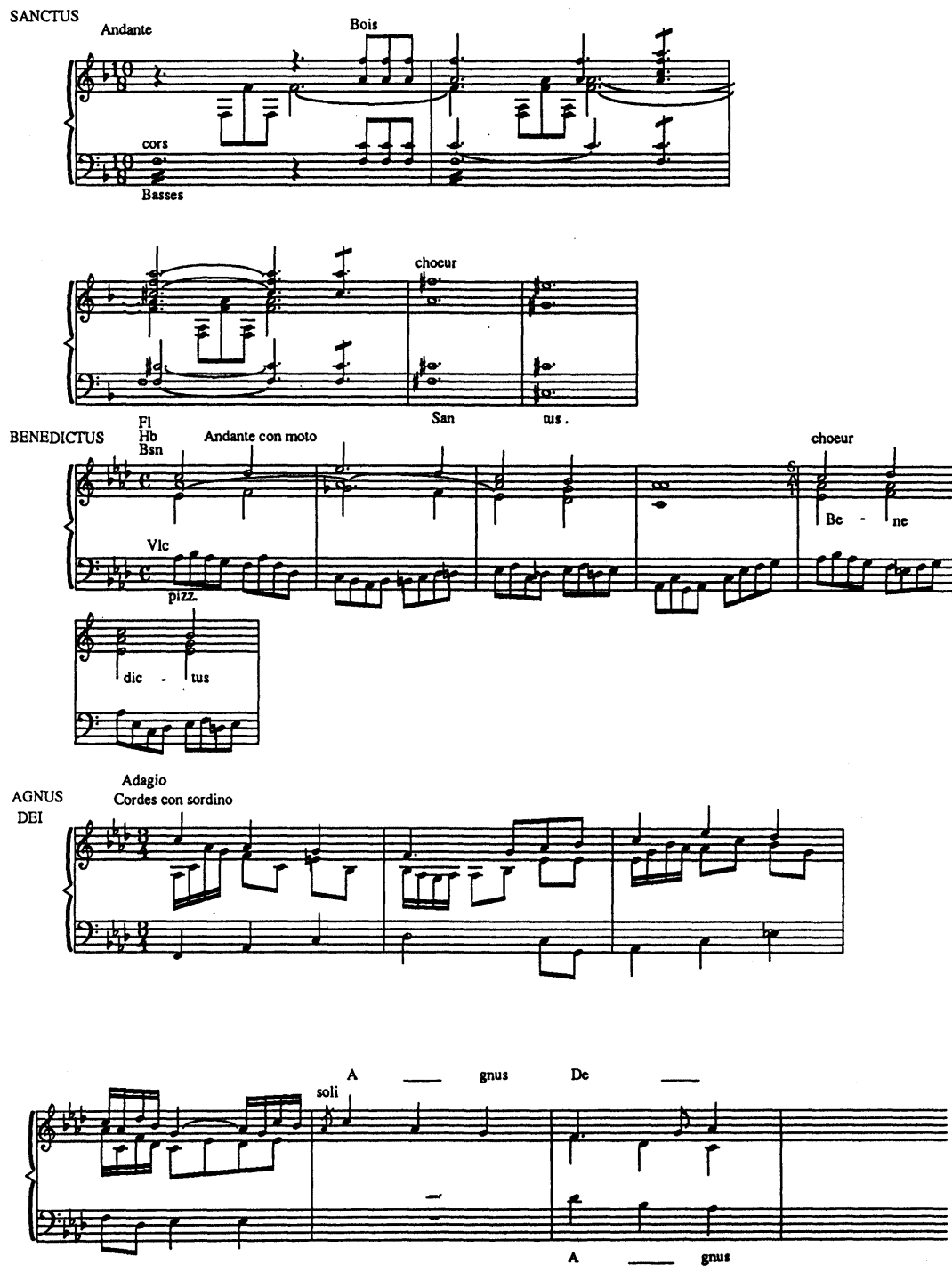

DONA

NOBIS Allegretto

PACEN

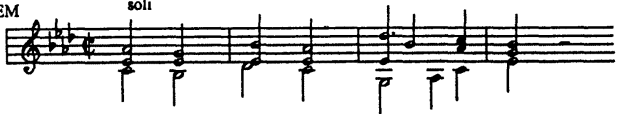

Do - na no - bis pa - - cern 
On remarque que les tonalités de la première colonne appartiennent à une même division de l'octave par la tierce majeure. La seule autre tonalité principale est $\mathrm{Fa}$ : ton relatif du ton principal de Lab, comme tel (Fa mineur) et avec considération du mode mixte ( $\mathrm{Fa}$ majeur).

Schubert avait déjà employé un procédé similaire dans un recueil d'Allemandes pour piano dont la date de composition, incertaine, est parfois estimée à $1812 !^{10}$ Postérieurement, soit dans ses 6 Bagatelles, op. 126 (1824), Beethoven aura recours au procédé, et pour retrouver celuici dans une cuvre d'envergure, il faut attendre la Première Symphonie, op. 68, de Brahms (1877).

\subsection{Tonalités importantes d'un mouvement}

Examinons le "Menuetto" du Quatuor en la mineur, D. 804, op. 29 (1824) - voir exemple 6.

Ce mouvement est en $\mathrm{La}$ mineur, et il est de forme ternaire A-B-A'. La partie A débute en $\mathrm{La}$ mineur, sur une pédale de dominante " $m i$ ", et se termine en Do majeur, tonalité relative, sur une pédale de " $d o$ ". Il semble exister ici une sorte de contradiction : les tonalités sont La et Do, mais les pédales sont " $m i$ " et " $d o$ ". L'usage de pédales de nature différente est un moyen privilégié par Schubert pour camoufler l'emploi $d^{\prime}$ une division de l'octave en parties égales. Parfois, $c^{\prime}$ est comme nous venons de le décrire, parfois c'est l'inverse : les tonalités sont en rapport de tierce majeure, mais non les pédales.

Avec "mi" et "do", il ne manque que "lab/sol\#" pour compléter la division de l'octave en tierces majeures. Or, la partie B (m. 21 et ss.), commence avec un accord de septième diminuée qui s'avère avoir comme fonction $\mathrm{V} / \mathrm{V}$ en $L a b$ majeur, la nouvelle tonique n'arrivant qu'à la mesure 28. Au dernier moment, Lab devient mineur et est suivi de la fonction VIb, qui n'est autre que l'accord de $M i$ majeur, dominante principale (m. 34-35). Il n'y a plus qu'à attendre le retour de $\mathrm{A}$, suite à cet habituel arrêt sur la dominante principale qui annonce la récapitulation à venir.

10 Il s'agit des 12 Wiener Deutsche, D. 128 (1812 ou 15). 

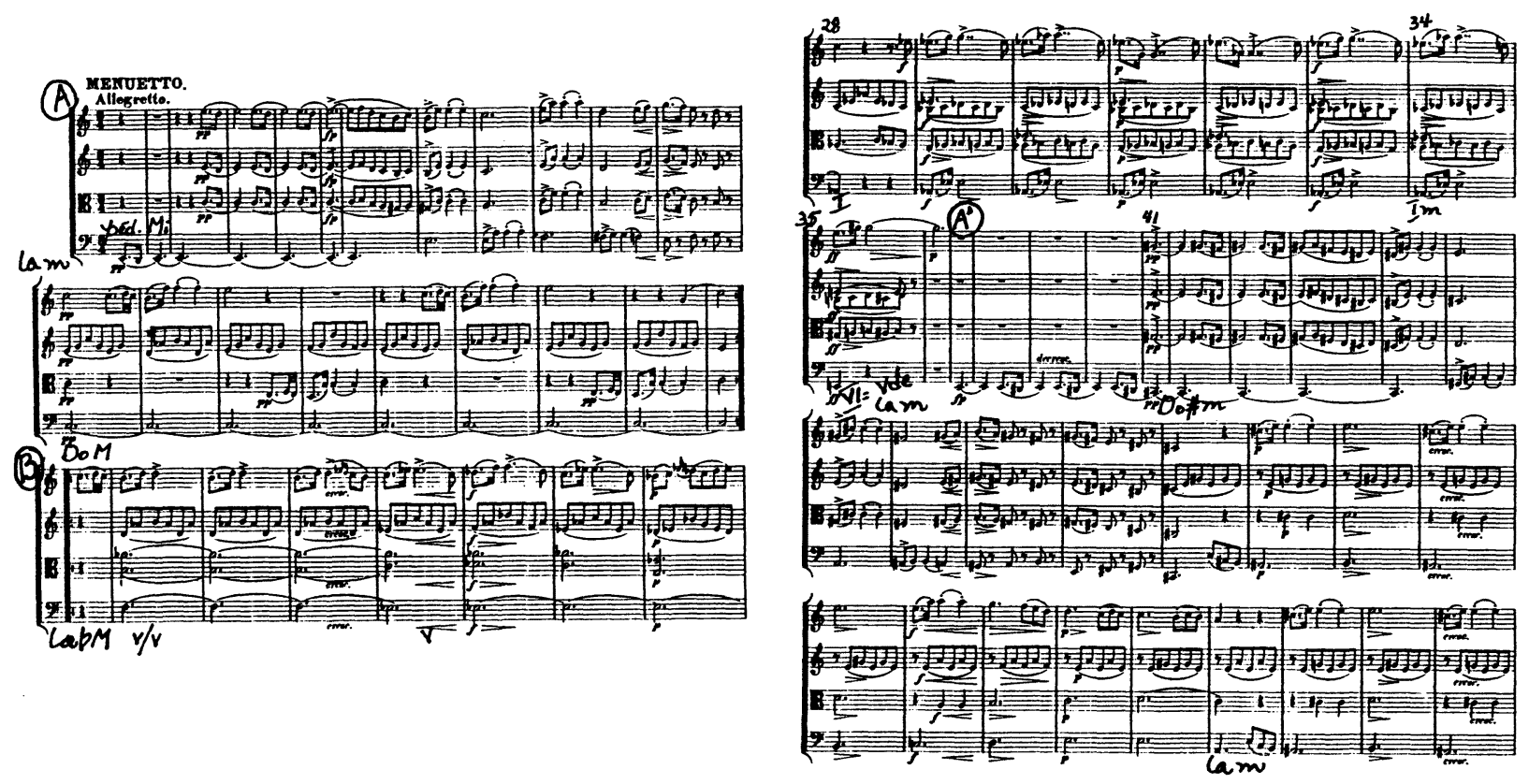

Exemple 6 : Schubert : Quatuor en La mineur, D.804, op. 29, "Menuetto" 
Nous avons ainsi une division symétrique de l'octave avec des éléments de diverse nature : Do est une tonalité importante, Lab est une tonalité provisoire, $M i$ est une pédale de dominante au début de A et la dominante sur laquelle aboutit $B$. Ce $n^{\prime}$ est pas tout. La partie $A^{\prime}$ ne se déroule pas tout à fait comme prévu : si, à l'instar de $\mathrm{A}$, elle débute par la note "mi" au violoncelle (m. 37), au dernier moment, une pirouette fait revenir le thème de $\mathrm{A}$ en Do\# mineur (m. 41 et ss.). Avec La mineur, ton principal, et Do\#, il ne manquerait que $\mathrm{Fa}$ pour compléter la division de l'octave en tierces majeures. Mais ici, la symétrie demeure partielle. L'idée d'une symétrie partielle est discutable : nous la relevons, dans ce cas, parce que Schubert a choisi Do\# plutôt que Fa. $\mathrm{Fa}$ fait partie du ton de $\mathrm{La}$ mineur, alors que Do\# vient contredire le fait mineur de La. Le rapport de tierce majeure entre La et Do\# est particulièrement saillant dans le contexte.

Globalement, nous avons une symétrie partielle au niveau de la tonique (division de l'octave en tierces majeures, incluant la tonique), alors que la symétrie est complète au niveau de la dominante (cette symétrie intègre notamment le relatif majeur). En principe, une symétrie au niveau de la dominante devrait être exclue des extrémités d'un mouvement d'œuvre, où l'on trouve habituellement la tonalité de tonique : Schubert remédie à la situation par l'emploi, dès le départ, $\mathrm{d}^{\prime}$ une pédale de dominante qui lui permet d'instaurer aussitôt sa division de l'octave en tierces majeures.

\subsection{Tonalités importantes d'une section d'une æuvre}

L'exemple choisi n'est pas si loin du précédent quant à son format. Il s'agit de la première partie de la Fantaisie en fa mineur, D. 940, op. 103, pour piano quatre mains (1828) - voir exemple 7. Comme la Wanderer-Fantasie, voilà une œuvre en un seul grand mouvement, mais dont les changements de tempo suggèrent fortement les quatre mouvements habituels. La première partie de la fantaisie est donc une espèce de mouvement miniature.

Le premier thème est de forme ternaire $\mathrm{A}-\mathrm{B}-\mathrm{A}^{\prime}$, en $\mathrm{Fa}$ mineur pour $\mathrm{A}$, et majeur pour $\mathbf{A}^{\prime}$. $\mathbf{A}^{\prime}$ est interrompu, soudainement, par un nouveau motif en $\mathrm{Fa}$ redevenu mineur (m. 48). Un enchaînement surprenant, 
48
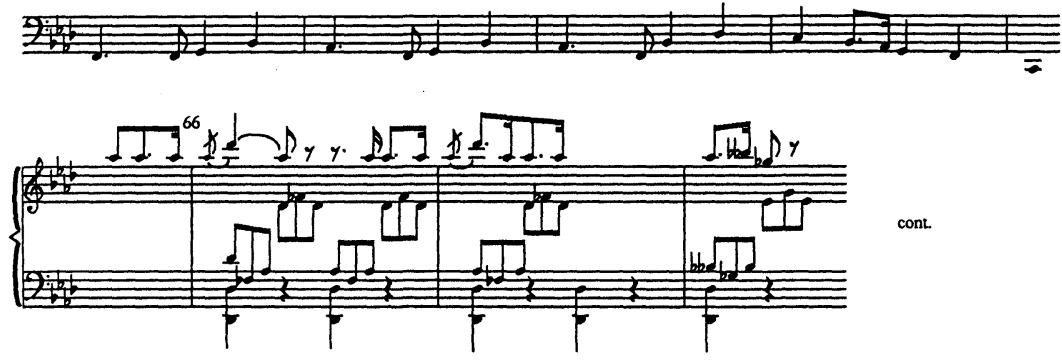

$\pi^{22}+2+2+2$

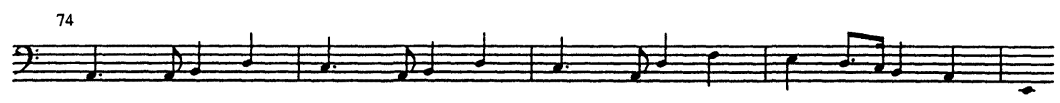

8va
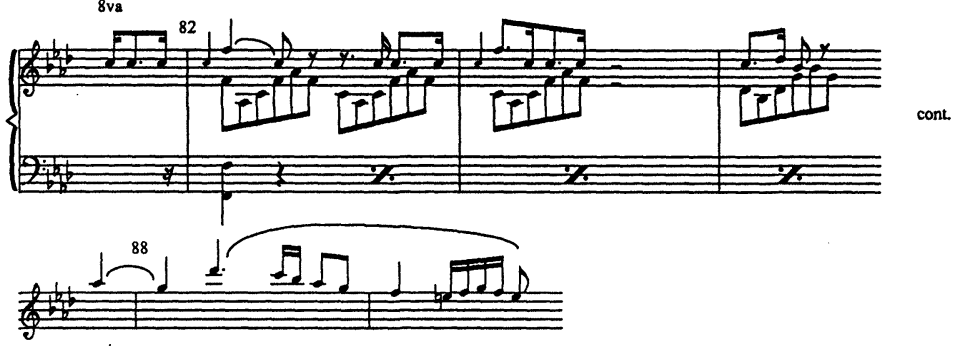

Exemple 7 : Schubert : Fantaisie en Fa mineur, D.940, op. 103, pour piano quatre mains 
V-VI mineur, ramène le motif initial en Réb mineur (m. 66). Celui-ci bifurque, au dernier moment, pour laisser la place au motif apparu mes. 48, maintenant en $L a$ mineur ( $m$. 74). De la même manière que l'on passait, précédemment, de $F a$ mineur à $R e ́ b$ mineur, on passe ici de $L a$ mineur à $F a$ mineur, avec le retour du motif initial (m. 82). Ce dernier se termine comme la toute première fois et la première partie prend fin avec une codetta en $\mathrm{Fa}$ majeur. On remarque que les tonalités présentes dans cette première partie appartiennent à une division de l'octave en tierces majeures au niveau de la tonique $(F a-R e ́ b-L a-F a)$. La dernière arrivée de $F a$ mineur s'est faite subtilement, dans le cadre d'une marche, effet comparable à celui d'une récapitulation amenée par un "faux retour".

\subsection{Tonalités provisoires}

La marche d'harmonie modulante sera privilégiée par Schubert lors de l'emploi de la division de l'octave en tierces majeures. L'exemple que voici est probant à cet égard. Il s'agit d'un extrait du premier mouvement de la Symphonie Tragique, D. 417 (1816), et, plus précisément, de la continuation du deuxième thème - voir exemple 8 .

Le deuxième thème, en $\mathrm{La}$ majeur, est entendu deux fois (m. 67-75 et 76- 84). Nous avons par la suite le modèle d'une marche basée sur la cellule initiale de ce thème $(\mathrm{m} .67$ et ss.). Ce modèle est modulant, la cellule répétée à la basse sur harmonie chromatique débute en Lab majeur (m. 85) et se dirige vers un tutti arpégé en $M i$ majeur (m. 89-93). La modulation s'est opérée en vertu du mode mixte : IV mineur en Lab majeur = VI en $M i$ majeur, par enharmonie. La fin du tutti provoque une élision avec la première reprise transposée du modèle; la modulation se fait maintenant de $M i$ majeur à $D o$ majeur (m. 93-101). Une deuxième reprise entraîne une modulation de $D o$ majeur à $L a b$ majeur (m. 101-109), pour un retour à la tonalité de départ. L'exposition se poursuit en $L a b$ majeur et elle se termine quand $L a b$ devient approche de la dominante du ton principal de Do mineur.

Dans cet exemple, la marche se compose d'un modèle répété deux fois, ce qui est courant. On remarquera toutefois que les répétitions correspondent à des transpositions exactes. Or, dans les marches diatoniques par tierces, le modèle est rarement tranposé exactement; on assiste plutôt à une alternance des modes majeur et mineur, alternance 
ลิ

theme

$-{ }^{85}{ }^{\text {Continuation) }}$

93 Bases

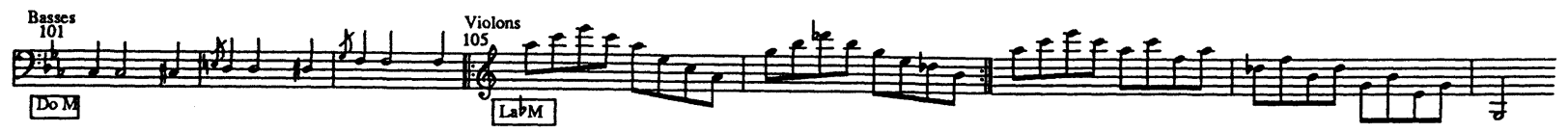

Exemple 8 : Schubert : Symphonie Tragique, D.417, I 
qui respecte l'armure. Si la musique dont il est ici question peut sembler redondante, de par la double répétition des mêmes mesures, tonalement, elle effectue un tour complet du cercle tonal. Comme dans l'exemple de la Fantaisie en Fa mineur (cf. point 3.3), nous sommes de retour à la tonalité de départ à notre grande surprise, puisqu'on avait l'impression de s'en éloigner. Enfin, soulignons que cette division se déroule en pleine exposition et non pas dans le développement, où des enchaînements tonals inhabituels sont plus courants. ${ }^{11}$

\subsection{Symétrie complète en variation d'une symétrie partielle}

Nous avons vu l'idée d'une symétrie partielle avec l'exemple du Quatuor en La mineur (point 3.2) : on y trouvait deux des trois éléments nécessaires à une symétrie complète. Dans l'exemple qui suit, nous avons une symétrie partielle dans la section $A$, puis complète dans $A^{\prime}$.

Il s'agit du deuxième mouvement de la Sonate en Sib, D. 960 (1828) voir exemple 9. Ce mouvement est en Do\# mineur et de forme ternaire A-B-A'. La section A se déroule ainsi :

- idée de base en Do\# mineur

(pédale de tonique "do\#")

- continuation sur pédale de dominante "sol\#" $\quad-$ m. 9-13

- cadence sur $M i$ majeur

Le thème est alors repris dans une version majeure en $M i$ :

- idée de base en $M i$ majeur

(pédale inférieure de tonique "mi", pédale supérieure de dominante "si")

- continuation sur pédale de dominante, mouvement "rompu" (V-VI) ramenant soudainement le ton de Do\# mineur pour une codetta

La symétrie partielle met en présence la pédale de dominante "sol\#" avec la tonique qui suit, $M i$. À vrai dire, il n'y a pas lieu, à ce moment-ci de la pièce, de relever cette symétrie, mais c'est à la lueur

11 Seidel signale cet exemple (1963:67). 
$\bar{\Xi}$

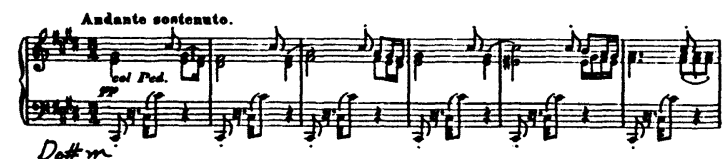

(2)

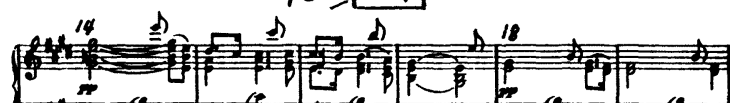

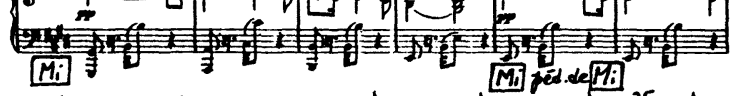

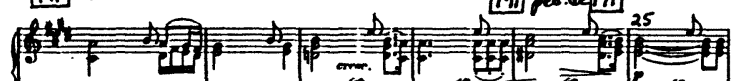

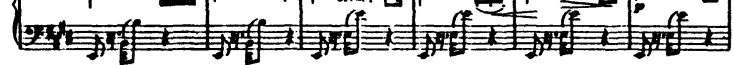

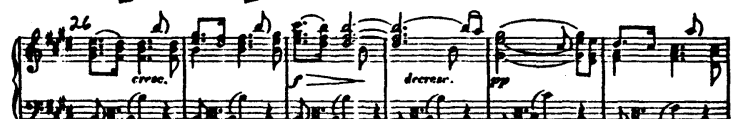

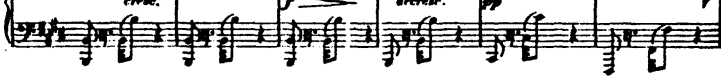

$[\cdots]$

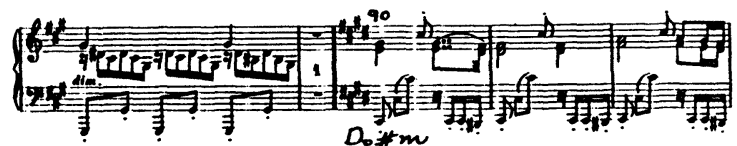

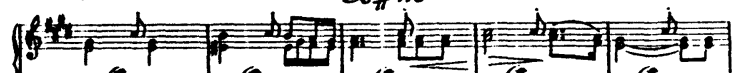

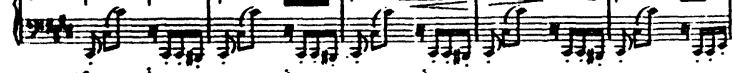

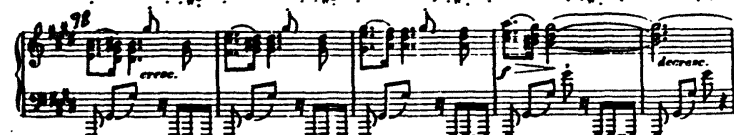

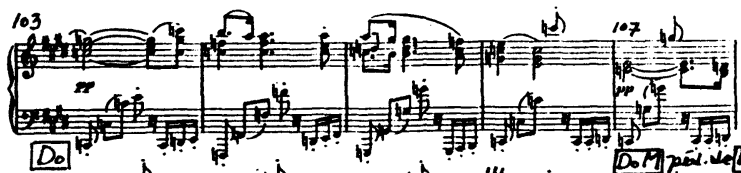
(20)

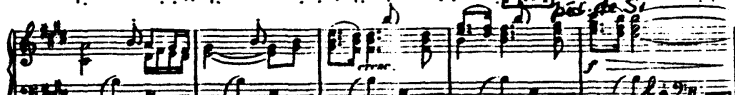

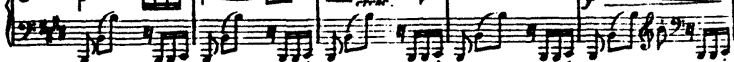

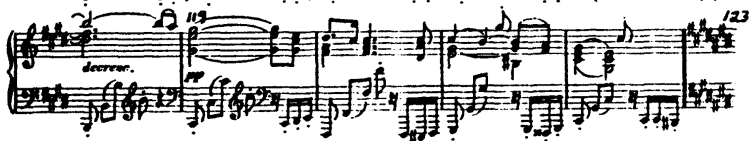

Exemple 9 : Schubert : Sonate en $\mathrm{Si}$ majeur, D.960, II 
de ce qui se produit dans la section $\mathrm{A}^{\prime}$ que nous nous permettons de le faire. La section $A^{\prime}$ se déroule ainsi :

idée de base en Do\# mineur

continuation sur pédale de "sol\#"

cadence sur Do majeur

Puis, la version majeure, cette fois en Do :

idée de base en Do majeur

- m. $107-110$

(pédale inférieure de tonique " $d o$ " pédale supérieure de $-\mathrm{m} .111-114$ idee de base reprise en $M i$ dominante "sol")

(pédales inférieure et supérieure de dominante "si") - m. 115- fin. continuation sur pédale de dominante "si", retour à Do\# comme dans A, coda en Do\# majeur

On voit bien, dans $\mathrm{A}^{\prime}$, une division de l'octave par tierces majeures, ascendantes cette fois, dont les éléments sont :

la pédale de dominante principale "sol\#"

le ton de Do majeur

le ton de $M i$ majeur

Les pédales sont respectivement "sol\#, $d o$, si" : Schubert a visiblement voulu camoufler la symétrie, en employant la pédale de dominante "si" pour le ton de $M i$ majeur dans $\mathrm{A}^{\prime}$, alors que dans $\mathrm{A}$, cette même tonalité se déroulait sur pédale de tonique "mi"! On pourrait voir une contradiction dans cette analyse : nous choisissons d'une part la pédale de "sol\#" dans le ton de Do\# mineur, et, d'autre part, le ton de $M i$ majeur malgré la pédale de "si". Nous considérons avoir le choix : la tonalité est toujours importante, mais une pédale ou encore, une insistance sur un degré d'une tonalité sont autant d'éléments qui attirent l'attention et méritent d'être considérés. Et c'est cette liberté de choix qui nous permet d'apprécier le jeu auquel Schubert s'est livré et de quelle façon il a cherché à brouiller les pistes. Qui aurait pu penser que l'accord de Do majeur (m. 103), climax du mouvement, était le résultat $\mathrm{d}^{\prime}$ une machination mathématique? Le grand art de Schubert consiste en ce que, à l'audition de sa musique, les mathématiques sont fort loin. 


\subsection{Toniques secondaires : emploi d'une gamme par tons harmonisée}

L'emploi de toniques secondaires en rapport de tierces majeures descendantes, dans le contexte où ces toniques sont précédées de leur dominante respective, permet d'obtenir deux gammes par tons différentes, qui se trouvent ainsi à être harmonisées. La première est obtenue si la quinte des accords de dominante secondaire se dirige vers la tonique des accords de tonique secondaire. La deuxième gamme par tons exige que les toniques secondaires soient mineures, et advient quand la septième des accords de dominante se dirige vers la tierce mineure des accords de tonique (voir exemple 10).

Ce phénomène survient à deux reprises dans le développement du premier mouvement de la Symphonie en Do, D. 944 (1825/28) - voir exemple 11. Lors de la première occurrence, on perçoit le déplacement sensible-tonique aux basses (m. 304 et ss.). On peut donc remarquer les couples V-I sur Lab mineur (m. 304-307), Mi mineur (m. 308-311) et Do mineur (m. 312-315), avant le retour à $L a b$ majeur pour une pédale de 12 mesures (m. 316-327). ${ }^{12}$ Les trombones ressortent avec leur motif qui est constitué du déplacement de la fondamentale vers la tierce de chacun des accords en présence. ${ }^{13}$ Les violons et les bois font entendre les deux gammes par tons à distance de tierce. Les deux gammes ne sont pas simultanées; les tierces surviennent suite à un frottement de seconde qui attire l'attention et dissimule ainsi les deux gammes par tons.

Après la pédale de 12 mesures, la même musique est reprise avec une nouvelle orchestration (m. 328-340). Violons et bois reprennent de la même manière leur double gamme par tons, mais une octave plus bas. Alti-bassons et violoncelles-contrebasses se partagent le motif des trombones; signalons que les notes des alti-bassons sont celles d'une nouvelle gamme par tons. Chacune des toniques secondaires reçoit une couleur particulière, puisque le mouvement mélodique "5-4-3-2-1" est entendu : à la flûte en Lab mineur (m. 329-331), au hautbois en $M i$ mineur (m. 333-335) et, enfin, à la clarinette en Do mineur (m. 337-339). Le développement se poursuit en Lab majeur (m. 340 et ss.).

12 Ce mouvement fourmille de symétries de tierces majeures apparaissant à différents niveaux. Signalons la symétrie qui se produit au niveau des tonalités importantes du mouvement : celui-ci est en $D o$ majeur à ses début et fin, le deuxième thème est en $M i$ mineur dans l'exposition, et le développement est principalement en Lab.

13 Ce motif des trombones est déjà présent à l'intérieur de l'exposition (m. 199 et ss.), dans un contexte où deux divisions de l'octave en tierces majeures se succèdent. 


\section{Exemple 10}

A $4 \hat{4} \quad 4 \hat{3} \quad \hat{4} \hat{3}$

\begin{tabular}{|c|c|c|c|}
\hline $2=$ & 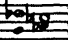 & & 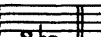 \\
\hline & $\hat{2} \hat{\imath}$ & $2 \hat{\imath}$ & 4 \\
\hline & 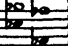 & $=$ & $\sigma$ \\
\hline & $\frac{\mathrm{L}_{\mathrm{b}}}{\mathrm{V}-\mathrm{I}}$ & $\frac{\mathrm{Mr} \text { m }}{\mathrm{V}-1}$ & $\frac{\overline{D o m}}{\frac{D o m}{V-1}}$ \\
\hline
\end{tabular}

Exemple 11 : Schubert : Symphonie en Do, D.944, I

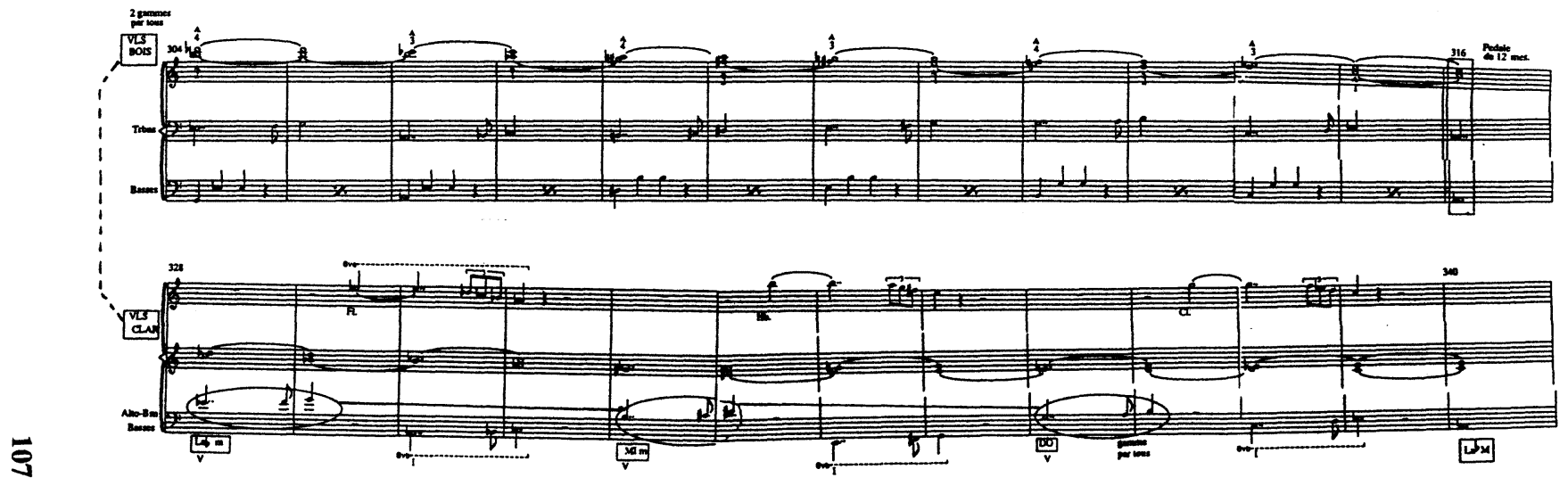




\subsection{Division par certains éléments ornementaux}

Examinons la fin du "Scherzo" du Quatuor en Sol , D. 887, op. 161 (1826) - voir exemple 12. La tonalité est $\mathrm{Si}$ mineur et nous pouvons observer que l'accord de tonique $S i$ est orné, harmoniquement, par un accord de sixte allemande reposant sur un "sol" (m. 126-130). Ce dernier accord agit habituellement comme approche de la dominante, mais ici, il ne fait qu'orner la quinte de l'accord de tonique. Jusqu'à présent, rien d'inquiétant : la sonorité de cette sixte allemande demeure familière. Schubert décide toutefois $\mathrm{d}^{\prime}$ orner de la même manière la tierce de l'accord de tonique "ré", soit par une sixte allemande reposant sur un " $m i$ " (m. 130-132), avant de poursuivre par de rassurants V-I (m. 132-136) et de reprendre le tout (m. 136 à la fin).

Nous avons donc un accord de Si mineur orné par des sixtes allemandes sur "sol" et " $m i$ ". L'accord sur " $m i$ " est tout à fait étonnant à l'audition, et on notera que l'orthographe indique bien "sib" pour cet accord, plutôt qu'un "la\#" sensible principale : il semble donc que Schubert voulait la sonorité d'un accord de Mib pour compléter la symétrie. ${ }^{14}$

\subsection{Accords consécutifs}

Un enchaînement d'accords dont les fondamentales sont à distance de tierce majeure descendante peut être plus ou moins courant. Si nous prenons l'exemple de l'enchaînement d'un accord de Do avec un accord de Lab, on conviendra que l'enchaînement Do mineur-Lab majeur est naturel (I-VI en Do mineur), alors que l'enchaînement Do majeur-Lab mineur s'explique difficilement dans le cadre $d^{\prime}$ une seule tonalité. L'addition d'un accord de $\mathrm{Mi}$, pour compléter la division symétrique de l'octave en tierces, donne nécessairement lieu à des enchaînements peu fréquents. Le moins que l'on puisse dire, c'est qu'un enchaînement d'accords Do-Lab-Mi-Do ne laisse pas à la tonalité le soin de l'enrober: la division l'octave est à découvert et nous nous trouvons momentanément en pleine atonalité.

14 Lors même qu'un accord de sixte allemande agit comme $\mathrm{V} / \mathrm{V}$, nous lui accordons deux fondamentales : 1) la fondamentale fonctionnelle, qui fait comprendre la fonction $\mathrm{V} / \mathrm{V}$ comme un accord de neuvième mineure de dominante, avec quinte abaissée et fondamentale tacite; 2) la fondamentale perçue à l'audition, soit la note de basse, "entendue" comme fondamentale $\mathrm{d}^{\prime}$ un accord de septième de dominante. C'est cette deuxième voie que nous avons choisie pour identifier les fondamentales dans l'exemple du point 3.7. 

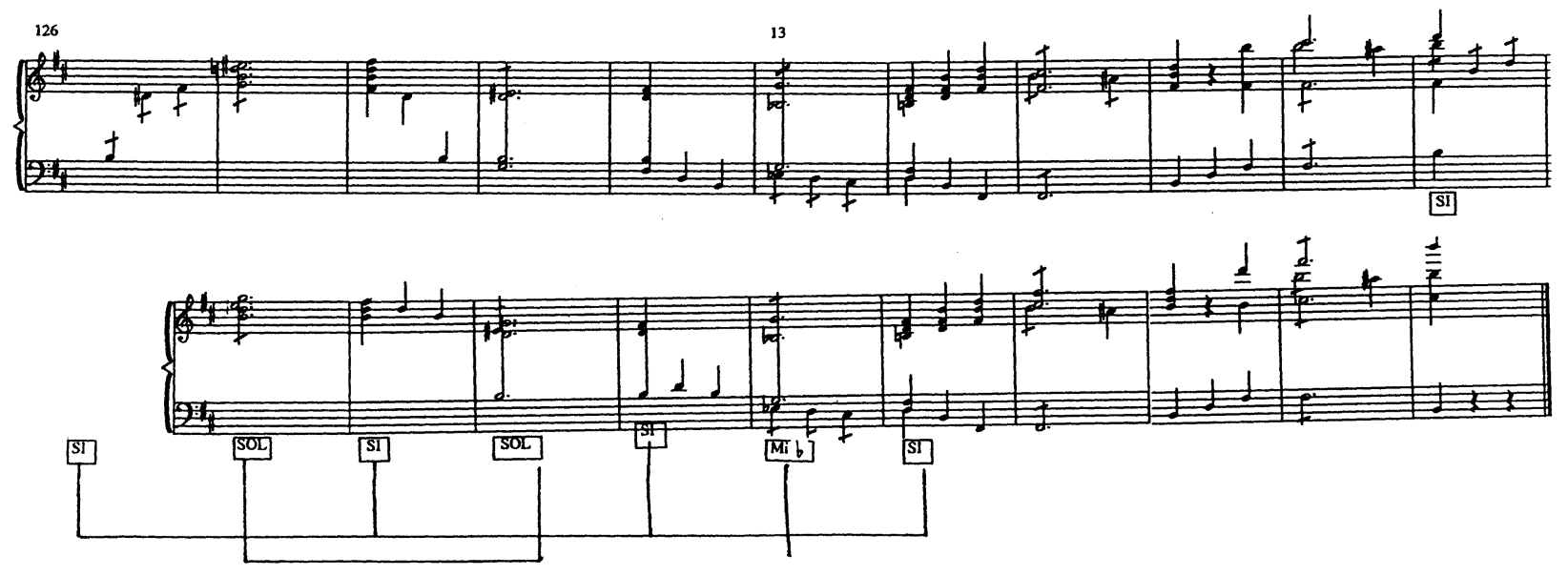

Exemple 12 : Schubert : Quatuor en Sol, D.887,ap.161, III 
Ce cas se produit, de manière évidente, au début du Sanctus de la Messe en Mib, D. 950 (1828) - voir exemple 13. Les trois "sanctus" interviennent respectivement sur des accords de $M i$ b majeur, $S i$ mineur et Sol mineur (m. 1-6). Ils sont suivis d'une formule cadentielle empruntée au mineur, formule qui s'amorce par des accords de Mib mineur et de Dob majeur (m. 7). ${ }^{15}$ Tous ces accords sont à l'état fondamental, les violoncelles et contrebasses articulant le ton qui sépare les fondamentales : le résultat est une gamme par tons mélodique dépassant l'octave. À nouveau, Schubert évite de répéter des accords de manière identique : ainsi, $M$ ib est d'abord majeur, puis mineur, alors que $S i$ mineur devient $D o b$ majeur.

Le début de ce Sanctus est véritablement incroyable : 1'harmonie certes, mais aussi les notes répétées aux cordes, l'orchestration et les effets de dynamique, tout fait que nous sommes dans un autre monde que celui de la tonalité !16

15 L'enchaînement des accords "si mineur-sol mineur-mib mineur" est déjà présent, et à plusieurs reprises, dans le Gloria de cette même Messe (cf., notamment, m. 56-58). Le texte est, là aussi, composé de trois "louanges" dont les paroles diffèrent toutefois : "laudamus te, benedicimus te, adoramus te".

16 On retrouve également cet exemple chez Seidel (1963:75, 165-166), Badura-Skoda (1982 : 198-199) et Taruskin (1985: 84-86). Aucun ne mentionne toutefois la présence d'une partie de l'enchaînement dans le Gloria (cf. note précédente). 
Sanctus.
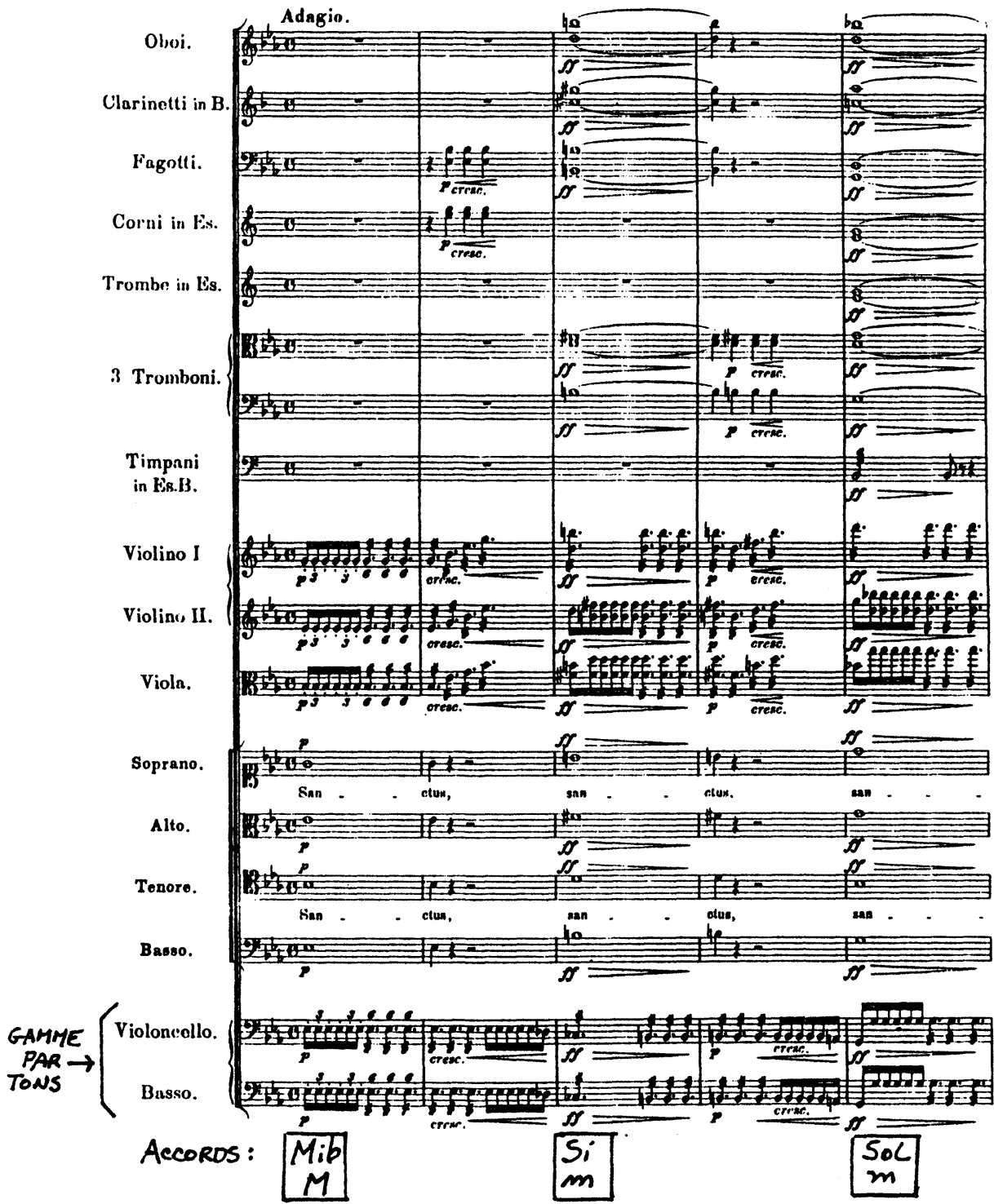

Exemple 13 : Schubert : Messe en Mi , D.950, "Sanctus" 


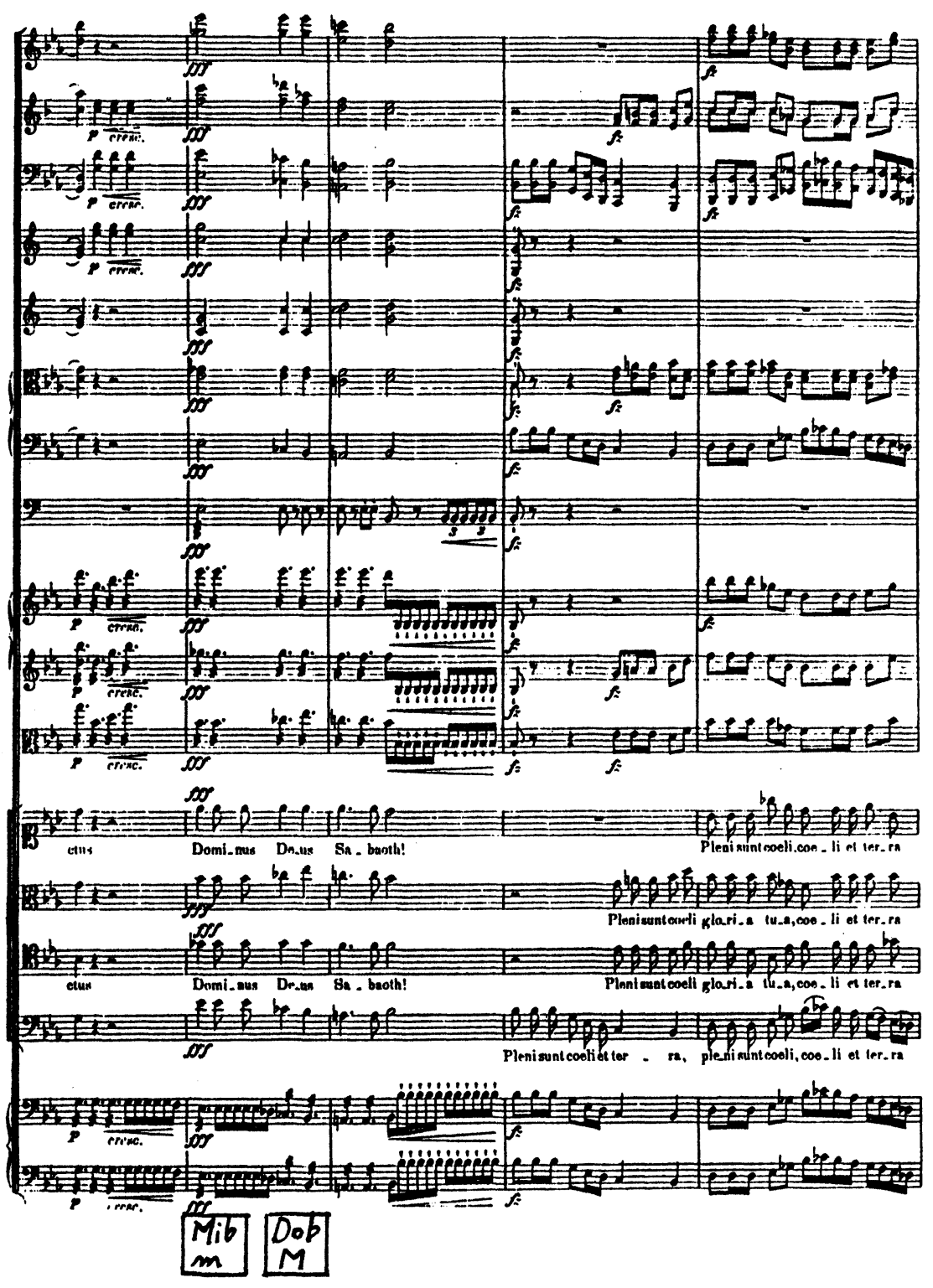

Exemple 13 : 


\section{Conclusion}

Les exemples ici étudiés démontrent clairement, croyons-nous, la volonté de Schubert de s'éloigner du diatonisme de la tonalité au profit du chromatisme impliquant tous les sons. Le chromatisme, qui contient le diatonisme, peut être tonal : les gammes chromatiques de Do majeur et de Do mineur ont une orthographe différente à cause des implications fonctionnelles de chacune des notes; ce sera le dodécaphonisme de Schœnberg qui fera disparaître définitivement cet aspect fonctionnel des 12 demi-tons. Mais le recours à la division symétrique d'une gamme chromatique contredit, en quelque sorte, le diatonisme et tend, par conséquent, à nous éloigner des habitudes tonales. Nous nous sommes limité, dans cet article, aux divisions symétriques de l'octave en parties égales et, plus précisément, à la division en trois tierces majeures, mais il est important de répéter que cette façon de faire n'en est qu'une parmi d'autres, ${ }^{17}$ privilégiée par Schubert, il est vrai.

L'enharmonie permet de considérer la tonalité comme circulaire; elle ouvre la voie au cercle tonal. Déjà, chez Bach, on peut trouver des "tournées" du cercle tonal, mais cela demeure exceptionnel. C'est Mozart et, surtout, Beethoven qui ont commencé à se servir de cette possibilité, bien que chez eux, les essais restent encore timides. Beethoven emploiera, par exemple, la division de l'octave en tierces majeures comme pôles tonals de certaines sections de "développement". Avec Schubert, le phénomène devient réel et il explique, en partie, ses "fameuses" modulations que l'on pouvait croire résultat de quelque hasard. Nous le retrouvons à tous les niveaux et cela, parfois, dans une même pièce. Le phénomène a de quoi étonner, d'autant plus que Schubert a tenté, la plupart du temps, de camoufler ses intentions. On doit reconnaître qu'il a bien réussi, puisqu'il a fallu énormément de temps pour que cet aspect tout à fait fondamental de son écriture soit mis en lumière. Notons, enfin, que l'emploi du procédé dans un contexte serré, comme celui des enchaînements d'accords, donne un résultat sonore que ses successeurs immédiats n'oseront même pas reproduire.

Schœnberg aurait déclaré : "On dit que je suis un révolutionnaire; je n'en suis qu'un bien petit en comparaison de Schubert."18 On peut certes

17 Pour un aperçu varié des procédés de symétrie employés par Schubert, cf. Seidel (1963).

18 Rapporté par Langevin (1979: 70). Ce qui nous intéresse ici n'est pas la 
interpréter cette phrase de diverses façons, mais nous sommes enclin à lui accorder le sens suivant : Schœnberg reçoit l'héritage tonal au moment où celui-ce se trouve dans une impasse et lui substitue un système nouveau, qui le renie complètement, alors que Schubert, en plein cour de l'ère tonale et tout en contribuant de manière importante à l'évolution de la tonalité, pose les premiers jalons de sa destruction. Or, le véritable miracle consiste en ce que, à l'audition, la musique de Schubert semble dans un tout autre monde que celui de la spéculation tonale! Son apparente simplicité ne devrait toutefois pas faire oublier qu'elle porte en germe une préfiguration de l'avenir.

\section{RÉFÉRENCES}

ALDWELL et SACHTER,

1979 : Harmony and Voice Leading 2. New York: Jovanovich.

BADURA-SKODA, Paul.

1982 : "Possibilities and Limitations of Stylistic Criticism in the Dating of Schubert's Great C Major Symphony", Schubert Studies (Cambridge University Press), 187-208.

BROWN, Maurice J.E.

1980 : "Schubert", The New Grove's Dictionary of Music and Musicians, vol. 16: 752-778.

DALHAUS, Carl.

1980 : "Harmony", The New Grove's Dictionary of Music and Musicians, Vol. 8: 175-188.

FEDERHOFER, Hellmut.

1978 : "Terzverwandte Akkorde und ihre Function in der harmonik Franz Schuberts", Schubert-Kongress 1978 (Graz: Akademische Druck und Verlagsanstalt), 61-70.

GOLDMAN, R.F.

1965 : Harmony in Western Music. New York : Norton.

LANGEVIN, P.G.

1979 : "Schubert après Schubert", L'Éducation musicale, 262, 67-71.

position humble adoptée par Schœnberg en regard de Schubert, mais plutôt le qualificatif révolutionnaire associé au nom du compositeur de l'époque romantique. 
LEBLANC, Mario.

1985 : Un trait fondamental de l'écriture harmonique de Franz Schubert : la division symétrique de l'octave en trois parties. Mémoire de maîtrise, Université McGill.

SCHENKER, Heinrich.

1979 : Free composition. New York : Longman.

SEIDEL, Elmar.

1963 : Die Enharmonik in der Grossformen Franz Schubert. Frankfurt : Gœthe Universität.

TARUSKIN, $R$.

1985 : "Chernomor to Kashchei : Harmonic Sorcery; or, Stravinsky's

'Angle'," Journal of the American Musicological Society, 38: 72-142.

WEBSTER, James A.

1978 : "The Schubert Sonata Form and Brahms first Maturity", 19th Century, 2: 18-35. 